\title{
A CONTRIBUTION TO MAGNETIC RECONNECTION: A BOLTZMANN CORRECTION TO THE MAGNETIC INDUCTION EQUATION FOR FARADAY VORTEX TUBES
}

\author{
Vladimir L. Borsch*
}

\begin{abstract}
The Boltzmann correction to the Maxwell induction law for a moving medium filled with vortex tubes of Faraday has been implemented.
\end{abstract}

Key words: the magnetic induction law, the theory of molecular vortices.

2010 Mathematics Subject Classification: 74A05, 74A25, 74F15, 76B47, 78A25.

\section{Communicated by Prof. O. A. Prykhod'ko}

\begin{abstract}
... It is true that at one time those who speculated as to the causes of physical phenomena, were in the habit of accounting for each kind of action at a distance by means of a special æthereal fluid, whose function and property it was to produce these actions. They filled all space three and four times over with æthers of different kinds, the properties of which were invented merely to 'save appearances,' so that more rational enquirers were willing rather to accept not only Newton's definite law of attraction at a distance, but even the dogma of Cotes, that action at a distance is one of the primary properties of matter, and that no explanation can be more intelligible than this fact...

$<\ldots\rangle$

But in all of these theories the question naturally occurs: - If something is transmitted from one particle to another at a distance, what is its condition after it has left the one particle and before it has reached the other?... Hence all these theories lead to the conception of a medium in which the propagation takes place, and if we admit this medium as an hypothesis, I think it ought to occupy a prominent place in our investigations, and that we ought to endeavour to construct a mental representation of all the details of its action, and this has been my constant aim in this treatise. [36]
\end{abstract}

\section{Introduction}

Maxwell's equations are foundational to electromagnetic theory. They are the cornerstone of a myriad of technologies and are basic to the understanding of innumerable effects. Yet there are a few effects or phenomena that cannot be explained by the conventional Maxwell theory. [2]

The governing equations of ideal magnetohydrodynamics (IMHD) are resulted from coupling the Maxwell equations for ideal conductive medium and the Euler equations for ideal fluid. A constitutive part of the IMHD governing equations is the (magnetic) induction law

$$
\boldsymbol{B}_{t}=\nabla \times(\boldsymbol{u} \times \boldsymbol{B}),
$$

where $\boldsymbol{u}(\boldsymbol{x}, t)$ is the fluid velocity, $\boldsymbol{B}(\boldsymbol{x}, t)$ is the magnetic induction, $(\boldsymbol{x}, t)$ is an inertial Cartesian orthogonal frame of reference, and the lower index $t$ indicates the partial derivative with respect to $t$.

\footnotetext{
*Dept. of Differential Equations, Faculty of Mech \& Math, Oles Honchar Dnipro National University, 72, Gagarin av., Dnipro, 49010, Ukraine, bvl@dsu.dp.ua

(C) Vladimir L. Borsch, 2018.
} 
The Alfvén theorem [14,16,21,37] of IMHD implies that the following two important properties of the magnetic induction $\boldsymbol{B}$ hold. The first property is the conservation of the magnetic flux

$$
\Psi[\mathcal{L}(t)]=\iint_{\mathcal{S}(t)} \boldsymbol{\nu} \cdot \boldsymbol{B} \delta \mathcal{S},
$$

where $\mathcal{L}(t)$ is an arbitrary closed material (co-moving) contour, $\mathcal{S}(t)$ is a surface bounded by the contour $\mathcal{L}(t), \boldsymbol{\nu}(\boldsymbol{x}, t)$ is the unit vector normal to $\mathcal{S}(t), \delta$ is the (purely) 'spatial' differential (at time $t$ being constant). The property is proved directly

$$
\dot{\Psi}[\mathcal{L}(t)]=\frac{\mathrm{d}}{\mathrm{d} t} \iint_{\mathcal{S}(t)} \boldsymbol{\nu} \cdot \boldsymbol{B} \delta \mathcal{S}=\iint_{\mathcal{S}(t)} \boldsymbol{\nu} \cdot\left[\frac{\partial \boldsymbol{B}}{\partial t}-\nabla \times(\boldsymbol{u} \times \boldsymbol{B})\right] \delta \mathcal{S} \stackrel{(1.1)}{=} 0
$$

where dot over a symbol here and below indicates the material ('total') derivative with respect to $t$.

An other proof follows from the first Zorawski criterion [53]. It says that the necessary and sufficient condition for the flux of an arbitrary vector field $\boldsymbol{a}(\boldsymbol{x}, t)$ through the material surface $\mathcal{S}(t)$ to conserve reads

$$
\dot{\boldsymbol{a}}-\boldsymbol{a} \cdot \nabla \boldsymbol{u}+(\nabla \cdot \boldsymbol{u}) \boldsymbol{a}=\mathbf{0},
$$

or equivalently

$$
\boldsymbol{a}_{t}+\boldsymbol{u} \cdot \nabla \boldsymbol{a}-\boldsymbol{a} \cdot \nabla \boldsymbol{u}+(\nabla \cdot \boldsymbol{u}) \boldsymbol{a}=\mathbf{0} .
$$

Using the following well known vector identity

$$
\nabla \times(\boldsymbol{a} \times \boldsymbol{b})=\boldsymbol{b} \cdot \nabla \boldsymbol{a}-(\nabla \cdot \boldsymbol{a}) \boldsymbol{b}-\boldsymbol{a} \cdot \nabla \boldsymbol{b}+(\nabla \cdot \boldsymbol{b}) \boldsymbol{a},
$$

where $\boldsymbol{b}(\boldsymbol{x}, t)=\boldsymbol{u}(\boldsymbol{x}, t)$, we obtain the condition (1.5) to be

$$
\boldsymbol{a}_{t}-\nabla \times(\boldsymbol{u} \times \boldsymbol{a})+(\nabla \cdot \boldsymbol{a}) \boldsymbol{u}=\mathbf{0} .
$$

When $\boldsymbol{a}=\boldsymbol{B}, \nabla \cdot \boldsymbol{B}=0$ (see the fourth equation of the system (2.1) or the second equation of the system (2.3)), the condition (1.7) transforms into the induction law (1.1).

The second property is the magnetic field line conservation, that is the magnetic lines co-move with the fluid, or they are 'frozen' into the fluid. The second Zorawski criterion [53] says that the necessary and sufficient condition for the vector field $\boldsymbol{a}(\boldsymbol{x}, t)$ to be material reads

$$
\boldsymbol{a} \times\left[\boldsymbol{a}_{t}-\nabla \times(\boldsymbol{u} \times \boldsymbol{a})+(\nabla \cdot \boldsymbol{a}) \boldsymbol{u}\right]=\mathbf{0} .
$$

Again, if $\boldsymbol{a}=\boldsymbol{B}$, the above condition holds due to $\nabla \cdot \boldsymbol{B}=0$ and the induction law (1.1). 
The both Zorawski criteria are thoroughly discussed in $[42,48]$.

The second property of the magnetic induction is usually used to introduce the magnetic field line velocity $\boldsymbol{w}(\boldsymbol{x}, t)$ and to consider the induction equation (1.1) in the following formulation

$$
\boldsymbol{B}_{t}=\nabla \times(\boldsymbol{w} \times \boldsymbol{B}) .
$$

The component $\boldsymbol{w}_{\|}$of $\boldsymbol{w}$ in the direction of $\boldsymbol{B}$ is actually not determined because a one-to-one correspon dence between field lines does not require one-to-one correspondence between the individual points lying on them [41], whereas the component of $\boldsymbol{w}$ in the direction normal to $\boldsymbol{B}$ is $\boldsymbol{w}_{\perp}=\boldsymbol{u}_{\perp}$. However, the usual convention is to assume that $\boldsymbol{w}_{\|}=\boldsymbol{u}_{\|}$, that is $\boldsymbol{w}=\boldsymbol{u}$.

The above two properties of the magnetic induction $\boldsymbol{B}$ are exactly those known in ideal hydrodynamics (IHD) for the vorticity

$$
\boldsymbol{\Omega}=\nabla \times \boldsymbol{u}
$$

and derived from the Kelvin theorem [25] or the Helmholtz equation [19,25]

$$
\boldsymbol{\Omega}_{t}=\nabla \times(\boldsymbol{u} \times \boldsymbol{\Omega}) .
$$

In IHD phenomena when all the hypotheses of the Kelvin theorem meet then the property of the conservation of the vorticity lines holds. In contrast to IHD, in IMHD the magnetic topology may change even when all the hypotheses of the Alfvén theorem meet. A well known example of such a change is magnetic reconnection. The phenomenon occurs in the solar corona, the Earth's magnetosphere, and laboratory plasmas. Detailed surveys on the subject are presented in $[17,22,41,43,51,52]$. We note, just in case, that Barrett [2] set up a list of electromagnetic phenomena not explained by the Maxwell equations.

Since in most theories of magnetic reconnection the induction law (whether in ideal or non-ideal cases) plays an important role, our concern is the origin of the induction law in the IMHD limit, rather then magnetic reconnection itself.

The article is arranged as follows.

In section 2 we consider the Minkowski approach currently adopted as a 'standard' in most of the existing textbooks on MHD for deriving the Maxwell equations in moving media.

In section 3 we consider the Maxwell approach based on the theory of molecular vortices and some mechanical analogies to derive the induction law. One should refer to [46] to learn more about the theory of molecular vortices and to $[10,26$, 39] to know out much interesting on the Maxwell way of reasoning. We show that in contrast to the well known common opinion Maxwell himself derived the induction law not only for media at rest but for moving ones as well. In the IMHD limit his induction law is nothing but the induction law (1.1) of the IMHD. The history of electrodynamics of moving media is fundamentally surveyed by Darrigol [11-13]. 
In section 4 we consider some observations of Boltzmann concerning the Maxwell study on the subject. Boltzmann thoroughly studied the Maxwell legacy on electromagnetism, namely three articles [29], [30-33], [34], and the two-volume book $[35,36]$. He translated the first and the second articles in German $[4,5]$ and supplemented both translations with his own very detailed and insight comments. He also published in English [6] the list of faults found by him in the first article. We implement some comments of Boltzmann to the second article to derive the corrected induction law.

In section 5 we consider the induction law corrected by Hornig [20] to preserve the magnetic line topology and not to preserve the magnetic flux. The induction law after Hornig happens to include the induction law after Boltzmann as a particular case provided some conditions meet.

In section 6 we consider the analogy between the Kelvin and the Alfvèn theorems and their consequences once again. Some of quite recently published results of other authors, for example, by Tsinober [50], prove that the analogy is imperfect or even does not hold. We show that the induction law after Boltzmann does not actually obey the analogy.

In section 7 we list in brief our observations on the subject.

\section{The induction law after Minkowski}

Because of our incomplete knowledge of the structure of matter, however, we are entitled to ask ourselves what statements the relativity principle allows us to make concerning (macroscopic) processes in moving bodies, assuming processes in bodies at rest to be experimentally known. This question was answered by Minkowski... He showed that the equations for moving bodies follow unambiguously from the relativity principle and from Maxwell's equations for bodies at rest... [40]

We shall not use these formulae in the rather complicated form in which they can be found in Maxwell's treatise, but in the clearer and more condensed form that has been given them by Heaviside and Hertz. [28]

The "Maxwell's equations" of today are due to Heaviside's "redressing" of Maxwell's work, and should, more accurately, be known as the "Maxwell-Heaviside equations." Essentially, Heaviside took the twenty equations of Maxwell and reduced them to the four now known as "Maxwell's equations." [2]

The governing equations of electromagnetism being actually the Hertz - Heaviside ones but usually attributed to Maxwell in the proper inertial Cartesian orthogonal frame of reference $\left(\boldsymbol{x}^{\prime}, t^{\prime}\right)$, where an undeformable conductive medium is at rest, read $[14,16,21,40]$

$$
\left\{\begin{array}{l}
\nabla^{\prime} \times \boldsymbol{H}^{\prime}=+\boldsymbol{D}_{t^{\prime}}^{\prime}+\boldsymbol{j}^{\prime}, \\
\nabla^{\prime} \times \boldsymbol{E}^{\prime}=-\boldsymbol{B}_{t^{\prime}}^{\prime}, \\
\nabla^{\prime} \cdot \boldsymbol{D}^{\prime}=q, \\
\nabla^{\prime} \cdot \boldsymbol{B}^{\prime}=0
\end{array}\right.
$$

where the following constitutive equations: $\boldsymbol{B}^{\prime}=\mu \boldsymbol{H}^{\prime}, \boldsymbol{D}^{\prime}=\varepsilon \boldsymbol{E}^{\prime}$, and the Ohm law $\boldsymbol{j}^{\prime}=\sigma \boldsymbol{E}^{\prime}$ are used; $\boldsymbol{E}$ and $\boldsymbol{H}$ being the electric and magnetic fields, $\boldsymbol{D}$ being 
the electric induction (displacement), $\varepsilon$ and $\mu$ being the electric and magnetic impermeabilities of the medium, $q$ being the volume density of the free electric charges, $\boldsymbol{j}$ being the surface density of the electric current, $\sigma$ being the conductivity of the medium.

For an undeformable moving medium in an inertial frame of reference $(\boldsymbol{x}, t)$ moving with the constant velocity $\boldsymbol{v}:|\boldsymbol{v}| \ll c$, where $c$ is the speed of light, with respect to the frame of reference $\left(\boldsymbol{x}^{\prime}, t^{\prime}\right): t^{\prime}=t, \boldsymbol{x}^{\prime}=\boldsymbol{x}-t \boldsymbol{v}$, the following nonrelativistic transformations of the dependent variables [40]

$$
\left\{\begin{aligned}
\boldsymbol{H}^{\prime} & =\boldsymbol{H}-\boldsymbol{v} \times \boldsymbol{D}, \\
\boldsymbol{E}^{\prime} & =\boldsymbol{E}+\boldsymbol{v} \times \boldsymbol{B}, \\
\boldsymbol{j}^{\prime} & =\boldsymbol{j}-\rho \boldsymbol{v}, \\
\rho^{\prime} & =\rho+\varepsilon \nabla \cdot(\boldsymbol{v} \times \boldsymbol{B}),
\end{aligned}\right.
$$

are used for the system of equations (2.1) to hold.

In the IMHD limit the above transformations simplify to the following ones

$$
\left\{\begin{array}{l}
\boldsymbol{H}^{\prime}=\boldsymbol{H}, \\
\boldsymbol{E}^{\prime}=\boldsymbol{E}+\boldsymbol{v} \times \boldsymbol{B}=\mathbf{0},
\end{array}\right.
$$

and the remaining part of the system (2.1) reads

$$
\left\{\begin{aligned}
\boldsymbol{B}_{t}-\nabla \times(\boldsymbol{v} \times \boldsymbol{B}) & =0, \\
\nabla \cdot \boldsymbol{B} & =0 .
\end{aligned}\right.
$$

Accounting for the vector identity (1.6), where $\boldsymbol{a}=\boldsymbol{v}, \boldsymbol{b}=\boldsymbol{B}$, the magnetic induction law (the first equation of the system (2.3)) simplifies as follows

$$
\boldsymbol{B}_{t}-\nabla \times(\boldsymbol{v} \times \boldsymbol{B})=\boldsymbol{B}_{t}+\boldsymbol{v} \cdot \nabla \boldsymbol{B} \equiv \dot{\boldsymbol{B}}=\mathbf{0} .
$$

The above equation means that if an undeformable conductive medium moves with constant velocity $\boldsymbol{v}$ the magnetic field $\boldsymbol{B}$ remains unaltered.

In case of a deformable medium it is usually assumed that there is a unique continuously differentiable transformation between laboratory $(\boldsymbol{x}, t)$ (or Eulerean) and material $(\boldsymbol{X}, t)$ (or Lagrangean) frames of reference

$$
\boldsymbol{x}=\boldsymbol{\Phi}(\boldsymbol{X}, t),
$$

referred to the law of motion. Actually, the transformation (2.5) is rarely known, and the solution to the following Cauchy problem

$$
\left\{\begin{array}{l}
\dot{\boldsymbol{x}}(t)=\boldsymbol{u}(\boldsymbol{x}, t), \\
\boldsymbol{x}(0)=\boldsymbol{X}
\end{array}\right.
$$


where $\boldsymbol{u}$ is the medium velocity, is implied by the law of motion.

Point-wise application of the transformations (2.2) at $\boldsymbol{v}=\boldsymbol{u}$ leads to the induction equation for the moving deformable medium as follows

$$
\boldsymbol{B}_{t}-\nabla \times(\boldsymbol{u} \times \boldsymbol{B})=\mathbf{0} .
$$

These formulae are rigorously valid only for uniformly moving bodies and, because of the additivity of the fields, also when several bodies are present which move uniformly with different velocities and are separated by vacuum regions. The approximation to which... are correct will generally be the better, the smaller the acceleration of the substance. [40]

Hence, obtaining the induction law after Minkowski implies supplementary assumptions not referred to by most of the textbooks. Sedov [44,45] studied applicability of these assumptions to moving media at large deformations.

\section{The induction law after Maxwell}

The consideration of the action of magnetism on polarized light leads, as we have seen, to the conclusion that in a medium under the action of magnetic force something belonging to the same mathematical class as an angular velocity, whose axis is in the direction of the magnetic force, forms a part of the phenomenon.

This angular velocity cannot be that of any portion of the medium of sensible dimensions rotating as a whole. We must therefore conceive the rotation to be that of very small portions of the medium, each rotating on its own axis. This is the hypothesis of molecular vortices. [36]

We shall suppose at present that all the vortices in any one part of the field are revolving in the same direction about axes nearly parallel, but that in passing from one part of the field to another, the direction of the axes, the velocity of rotation, and the density of the substance of the vortices are subject to change. [30]

Auch die Gleichungen, welche Maxwell hier für die electromagnetische Wirkung in bewegten medien aufstellt, hat Hertz anfangs übersehen. [5]

To derive the induction law (as a constitutive part of his set of the governing equations for the electromagnetic phenomena) Maxwell, firstly, introduced an inviscid continuum (or a medium, referred to the microfluid below) consisting of cylindrical vortices rotating as quasi-rigid bodies (prop. I [30], pp. 165-167), as shown in Fig. 1.

Secondly, Maxwell interpreted quantities used in electrodynamics as follows: $\mu$ (magnetic impermeability) being a value depending on the density of the microfluid and the position of the vortices (prop. I [30], pp. 165-167, prop. III [30], pp. 167-175), $\boldsymbol{E}$ (the electric field induced by free electric charges) being the force with what intermediate particles treated as free electric charges act on the vortices (prop. VII [31], pp. 288-289), and $\boldsymbol{H}$ (the magnetic field, the magnetic induction $\boldsymbol{B}=\mu \boldsymbol{H}$ ) being the following vector (prop. I [30], pp. 165-167, prop. III [30], pp. $167-175)$

$$
\boldsymbol{H}=w \boldsymbol{\tau}=r \omega \boldsymbol{\tau}=r \boldsymbol{\omega},
$$

where $r, w$, and $\boldsymbol{\omega}$ are denoted in Fig. 1,a.

In prop. VIII [31], pp. 289-291, Maxwell derived the induction law for the microfluid at rest (the second equation of the system (2.1)), i. e. for the case when 

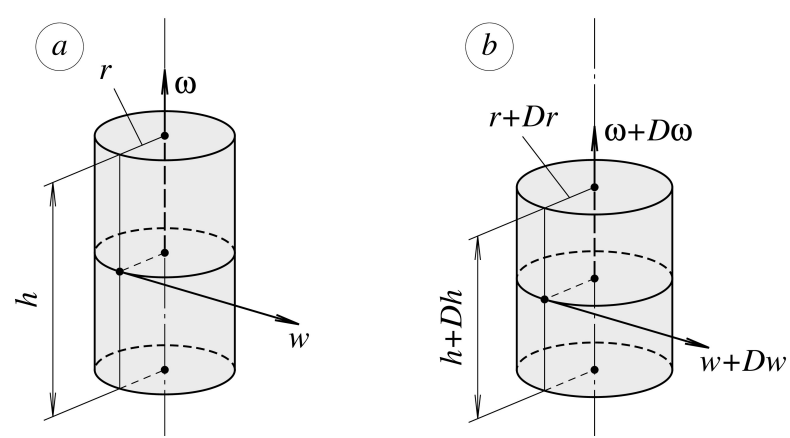

Fig. 1. Micromotion of the element of a vortex tube: before deformation at instant $t(a)$ and after deformation at instant $t^{\prime}(b)$

magnetic induction changes being influenced by the only field $\boldsymbol{E}$. In props. IX and $\mathrm{X}$ he considered the change of magnetic field being influenced by only small deformation, treating separately the strain and the rigid rotation between two instants $t$ and $t^{\prime}=t+\Delta t, \Delta t=\mathrm{d} t=\mathrm{D} t$.

In prop. IX [31], p. 340, Maxwell considered the infinitesimal 'parallelopiped' (not the infinitesimal cylinder, as the element of a vortex tube!) with its three edges being parallel to the axes $x_{1}, x_{2}, x_{3}$ of a fixed orthogonal frame of reference (or a Cartesian laboratory frame, see Fig. 2) and equaled to $h_{1}, h_{2}, h_{3}$. From the continuity property of the medium (this means that the volume of the parallelepiped remains unaltered) and the conservation of energy Maxwell concluded that due to the strain the following relations hold

$$
\frac{\mathrm{D}_{s t r} H_{\kappa}}{H_{\kappa}}=\frac{\mathrm{D}_{s t r} h_{\kappa}}{h_{\kappa}} \equiv \lambda_{\kappa}, \quad \kappa=1,2,3
$$

where D stands for the 'deformational' differential (one should not confuse the differential $\mathrm{D}$ with the differential $\delta$ in the magnetic flux definition (1.2) and the magnetic flux conservation property (1.3), since the differential $\delta$ is used only for the spatial integration, as the increment for spatial variables at $t$ being constant), $H_{\kappa}$ are the Cartesian components of the magnetic field $\boldsymbol{H}$, and $\lambda_{\kappa}$ are the extensions of the corresponding edges.

In the modern notation the above relations read

$$
\mathrm{D}_{s t r} \boldsymbol{H}=\boldsymbol{H} \cdot \hat{\boldsymbol{S}} \Delta t,
$$

where $\hat{\boldsymbol{S}} \Delta t$ is the symmetric tensor of small deformation, $\hat{\boldsymbol{S}}$ being the Euler stretching tensor [49].

In prop. X [31], pp. 340-341, Maxwell considered the rigid rotation of the 'parallelopiped' and derived the following equation (in the modern notation) 


$$
\mathrm{D}_{\text {rot }} \boldsymbol{H}=\boldsymbol{H} \cdot \hat{\boldsymbol{W}} \Delta t,
$$

where $\hat{\boldsymbol{W}} \Delta t$ is the skew-symmetric tensor of small rigid rotation, $\hat{\boldsymbol{W}}$ being the Cauchy spin tensor [49].

It is evident that the equations (3.3) and (3.4) are valid for the magnetic induction $\boldsymbol{B}$ being rewritten as follows

$$
\frac{\mathrm{D}_{s t r} \boldsymbol{B}}{\mathrm{D} t}=\boldsymbol{B} \cdot \hat{\boldsymbol{S}}, \quad \frac{\mathrm{D}_{r o t} \boldsymbol{B}}{\mathrm{D} t}=\boldsymbol{B} \cdot \hat{\boldsymbol{W}}, \quad \frac{\mathrm{D} \boldsymbol{B}}{\mathrm{D} t}=\boldsymbol{B} \cdot(\hat{\boldsymbol{S}}+\hat{\boldsymbol{W}}) .
$$

In prop. XI [31], pp. 341-348, Maxwell collected all the results obtained in props. VIII, IX, and X for the rates of change of $\boldsymbol{B}$ and equated the substantial derivative of $\boldsymbol{B}$ to the sum of the rates of change of $\boldsymbol{B}$ due to: 1) the action of the electric field $\boldsymbol{E}$, given by the second equation of the system $(2.1) ; 2)$ the strain, given by the first of the above equations; and 3) the rigid rotation, given by the second of the above equations, to obtain

$$
\dot{\boldsymbol{B}} \equiv \frac{\mathrm{d} \boldsymbol{B}}{\mathrm{d} t} \equiv \boldsymbol{B}_{t}+\boldsymbol{u} \cdot \nabla \boldsymbol{B}=\boldsymbol{B} \cdot \nabla \boldsymbol{u}-\nabla \times \boldsymbol{E},
$$

where the unique decomposition $\nabla \boldsymbol{u}=\hat{\boldsymbol{S}}+\hat{\boldsymbol{W}}[49]$ is applied.

Then Maxwell used the microfluid incompressibility condition $\nabla \cdot \boldsymbol{u}=0$ once again, the condition of 'the absence of free magnetism' $\nabla \cdot \boldsymbol{B}=0$ (Maxwell formulated this condition in terms of magnetic field, i. e. as $\nabla \cdot \boldsymbol{H}=0, \mu$ being constant), and the vector identity (1.6) to derive from the equation (3.5) the induction law for the moving microfluid

$$
\boldsymbol{B}_{t}=\nabla \times(\boldsymbol{B} \times \boldsymbol{u})-\nabla \times \boldsymbol{E} .
$$

The above equation was not aimed to be principal or final in the Maxwell theory and happened to be hidden in his calculations. Actually, Maxwell tried to account for the notion of electrotonic state introduced by Faraday [15].

The conception of such a quantity, on the changes of which, and not on its absolute magnitude, the induction current depends, occurred to Faraday at an early stage of his researches (Exp. Res., series I, 60)... He therefore recognised... a 'peculiar electrical condition of matter,' to which he gave the name of the Electrotonic State. He afterwards found that he could dispense with this idea by means of considerations founded on the lines of magnetic force (Exp. Res., series II, 242), but even in his latest researches (Exp. Res., series II, 3269), he says, 'Again and again the idea of an electrotonic state (Exp. Res., 60, 1114, 1661, 1729, 1733) has been forced upon my mind.' [36]

Central to the Maxwell formulation of electromagnetism was the Faraday concept of the electrotonic state (from the new Latin tonicus, "of tension or tone"; from the Greek tonos, "a stretching"). [2]

Hence, following the idea of electrotonic state, Maxwell introduced the vector potential $\boldsymbol{A}: \boldsymbol{B}=\nabla \times \boldsymbol{A}$, and derived from equation (3.6) the following one for the electric field

$$
\boldsymbol{E}=\boldsymbol{A}_{t}+\boldsymbol{u} \times \boldsymbol{B}+\nabla \varphi
$$


where $\varphi$ is a scalar potential.

Subsequently, the $\boldsymbol{A}$ field was banished from playing the central role in Maxwell's theory and relegated to being a mathematical (but not physical) auxiliary. This banishment took place during the interpretation of Maxwell's theory by the Maxwellians, i.e. chiefly by Heaviside, Fitzgerald, Lodge and Hertz. The "Maxwell theory" and "Maxwell's equations" we know today are really the interpretation of Maxwell by these Maxwellians. It was Heaviside who "murdered the $\boldsymbol{A}$ field" (Heaviside's description) and whose work influenced the crucial discussion which took place at the 1888 Bath meeting of the British Association (although Heaviside was not present). [2]

In the IMFD limit the induction law (3.6) of Maxwell reads

$$
\dot{\boldsymbol{B}}-\boldsymbol{B} \cdot \nabla \boldsymbol{u} \equiv \boldsymbol{B}_{t}+\boldsymbol{u} \cdot \nabla \boldsymbol{B}-\boldsymbol{B} \cdot \nabla \boldsymbol{u} \equiv \boldsymbol{B}_{t}+\nabla \times(\boldsymbol{u} \times \boldsymbol{B})=\mathbf{0} .
$$

\section{The induction law after Boltzmann}

Boltzmann, being an inquisitive and shrewd researcher of the Maxwell legacy, noticed (comment 39 to prop. IX [5], p. 114) that Maxwell had derived the induction equation (3.6) not accounting for the definition of the magnetic field (3.1) of his own. And it was Boltzmann who supplemented both the conditions used by Maxwell, the incompressibility one and the conservation of energy for the medium, with the condition of preserving the cylindrical shape of the vortex tubes to obtain the following correct constraints for the deformation of any element of the vortex tubes

$$
\frac{1}{2} \frac{\mathrm{D} \omega}{\omega}=\frac{\mathrm{D} w}{w}=-\frac{\mathrm{D} r}{r}=\frac{1}{2} \frac{\mathrm{D} h}{h} .
$$

Boltzmann showed that the corresponding Maxwell constraints were as follows

$$
\frac{\mathrm{D} \omega}{\omega}=\frac{\mathrm{D} w}{w}=-\frac{\mathrm{D} r}{r}=\frac{\mathrm{D} h}{h}
$$

and did not agree with preserving the cylindrical shape of the vortex tubes.

Unfortunately Boltzmann himself did not implement the constraints (4.1) and the Maxwell definition of the magnetic field (3.1) to derive the correct induction law. Hence, in what follows, we implement the Boltzmann correction.

For this we consider the material vector $\boldsymbol{h}=h \boldsymbol{\tau}=\boldsymbol{x}_{\mathrm{N}}-\boldsymbol{x}_{\mathrm{M}}$, determining the position of the vortex tube element MN at instant $t$ (Fig. 2,a). At instant $t^{\prime}$ the material vector transforms into $\boldsymbol{h}^{\prime}=h^{\prime} \boldsymbol{\tau}^{\prime}=\boldsymbol{x}_{\mathrm{N}^{\prime}}-\boldsymbol{x}_{\mathrm{M}^{\prime}}$ (Fig. 2, $b$ ), where $\boldsymbol{\tau}$ is the unit vector tangent to the axis of the element: $|\boldsymbol{\tau}|=\left|\boldsymbol{\tau}^{\prime}\right|=1$.

Using the law of motion of the medium (2.5) we represent the change of $\boldsymbol{h}$ through the material variables $\boldsymbol{X}$ and the time increment $\Delta t$ as follows

$$
\boldsymbol{h}^{\prime}-\boldsymbol{h}=\left[\mathbf{\Phi}\left(\boldsymbol{X}_{\mathrm{N}}, t^{\prime}\right)-\boldsymbol{\Phi}\left(\boldsymbol{X}_{\mathrm{M}}, t^{\prime}\right)\right]-\left[\boldsymbol{\Phi}\left(\boldsymbol{X}_{\mathrm{N}}, t\right)-\mathbf{\Phi}\left(\boldsymbol{X}_{\mathrm{M}}, t\right)\right],
$$

and consequently find that [49] 


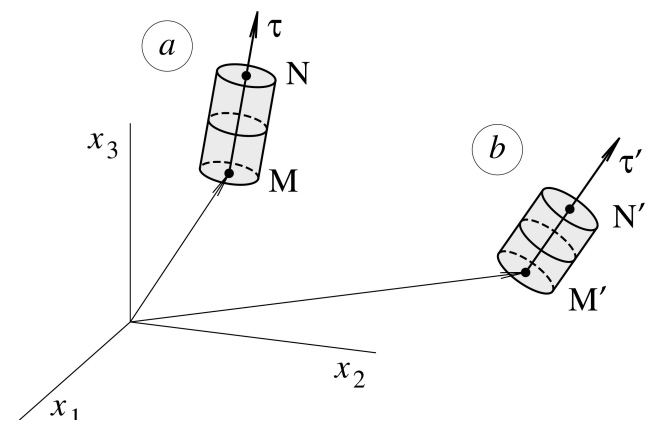

Fig. 2. Macromotion of the element MN of a vortex tube: before deformation at instant $t(a)$ and after deformation at instant $t^{\prime}(b)$

$$
\frac{\mathrm{D} \boldsymbol{h}}{\mathrm{D} t}=\lim _{\Delta \rightarrow 0} \frac{\boldsymbol{h}^{\prime}-\boldsymbol{h}}{\Delta t}=\boldsymbol{h} \cdot \nabla \boldsymbol{u},
$$

where the material variables $\boldsymbol{X}$ are assumed to coincide with the laboratory ones $\boldsymbol{x}$ at instant $t$ (see the formulation of the Cauchy problem (2.6)).

From the above relation for the 'deformational' time derivative of the material vector $\boldsymbol{h}$ we find for the squared length of $\boldsymbol{h}$

$$
\boldsymbol{h} \cdot \frac{\mathrm{D} \boldsymbol{h}}{\mathrm{D} t}=\frac{1}{2} \frac{\mathrm{D} \boldsymbol{h}^{2}}{\mathrm{D} t}=\frac{1}{2} \frac{\mathrm{D} h^{2}}{\mathrm{D} t}=h \frac{\mathrm{d} h}{\mathrm{~d} t}=\boldsymbol{h} \cdot(\hat{\boldsymbol{S}}+\hat{\boldsymbol{W}}) \cdot \boldsymbol{h}=\boldsymbol{h} \cdot \hat{\boldsymbol{S}} \cdot \boldsymbol{h}=h^{2} \theta
$$

where the scalar function

$$
\theta(\hat{\boldsymbol{S}}, \boldsymbol{h})=|\boldsymbol{h}|^{-2} \boldsymbol{h} \cdot \hat{\boldsymbol{S}} \cdot \boldsymbol{h}=\boldsymbol{\tau} \cdot \hat{\boldsymbol{S}} \cdot \boldsymbol{\tau}
$$

is the normal component of $\hat{\boldsymbol{S}}$ in the direction of the axis of the element.

Then, differentiating the definition of the magnetic field (3.1), we obtain

$$
\frac{\mathrm{D} \boldsymbol{H}}{\mathrm{D} t}=\frac{\mathrm{D} w}{\mathrm{D} t} \boldsymbol{\tau}+w \frac{\mathrm{D} \boldsymbol{\tau}}{\mathrm{D} t} \stackrel{(4.1)}{=} \frac{1}{2} \frac{w}{h} \frac{\mathrm{D} h}{\mathrm{D} t} \boldsymbol{\tau}+w \frac{\mathrm{D} \boldsymbol{\tau}}{\mathrm{D} t},
$$

where the logarithmic 'deformational' derivative of the length $h$ of the material vector $\boldsymbol{h}$ is already known from the equation (4.4) to be

$$
\frac{1}{h} \frac{\mathrm{D} h}{\mathrm{D} t}=\theta
$$

and the only derivative is needed to be find is the following one

$$
\frac{\mathrm{D} \boldsymbol{\tau}}{\mathrm{D} t}=\frac{\mathrm{D}}{\mathrm{D} t}\left(\frac{\boldsymbol{h}}{h}\right)=\frac{1}{h^{2}}\left(h \frac{\mathrm{D} \boldsymbol{h}}{\mathrm{D} t}-\frac{\mathrm{D} h}{\mathrm{D} t} \boldsymbol{h}\right) \stackrel{(4.3)}{=} \boldsymbol{\tau} \cdot \nabla \boldsymbol{u}-\theta \boldsymbol{\tau} .
$$


Substituting the 'deformational' derivatives of $\ln h(4.7)$ and $\boldsymbol{\tau}$ (4.8) into the right hand side of the equation (4.6) we find for the 'deformational' derivative of the magnetic field

$$
\frac{\mathrm{D} \boldsymbol{H}}{\mathrm{D} t}=\frac{1}{2} w \theta \boldsymbol{\tau}+w \boldsymbol{\tau} \cdot \nabla \boldsymbol{u}-w \theta \boldsymbol{\tau}=-\frac{1}{2} \theta \boldsymbol{H}+\boldsymbol{H} \cdot \nabla \boldsymbol{u} .
$$

The same equation is evident to hold for the magnetic induction

$$
\frac{\mathrm{D} \boldsymbol{B}}{\mathrm{D} t}=-\frac{1}{2} \theta \boldsymbol{B}+\boldsymbol{B} \cdot \nabla \boldsymbol{u} .
$$

Combining the 'deformational' derivative of $\boldsymbol{B}$ (4.9) with the time derivative of $\boldsymbol{B}$ due to the action of the electric field (the second equation of the system (2.1)) we obtain the induction law after Boltzmann

$$
\dot{\boldsymbol{B}} \equiv \frac{\mathrm{d} \boldsymbol{B}}{\mathrm{d} t}=\boldsymbol{B} \cdot \nabla \boldsymbol{u}-\frac{1}{2} \theta \boldsymbol{B}-\nabla \times \boldsymbol{E},
$$

and in the IMFD limit it reads

$$
\dot{\boldsymbol{B}} \equiv \frac{\mathrm{d} \boldsymbol{B}}{\mathrm{d} t}=\boldsymbol{B} \cdot \nabla \boldsymbol{u}-\frac{1}{2} \theta \boldsymbol{B} .
$$

Representing the total time derivative (material) at the left hand side of the above equation as the sum of the local and the convective derivatives we obtain the induction law in more usual formulation

$$
\boldsymbol{B}_{t}+\boldsymbol{u} \cdot \nabla \boldsymbol{B}-\boldsymbol{B} \cdot \nabla \boldsymbol{u}+\frac{1}{2} \theta \boldsymbol{B} \equiv \boldsymbol{B}_{t}+\nabla \times(\boldsymbol{B} \times \boldsymbol{u})+\frac{1}{2} \theta \boldsymbol{B}=\mathbf{0}
$$

\section{The induction law after Hornig}

\footnotetext{
Besides, some models are based not on a solution of the corresponding MHD equations but on some geometrical consideration and on ideas about the motion of frozen-in magnetic field lines. This concept of magnetic field line motion has often led to some confusion; because of that, some models based on that concept were accurately criticized by Alfvén $(1976,1977)$. We also believe that physical models cannot be based on the qualitative and to some degree speculative ideas on magnetic field line motion (the more so because in some regions the frozen-in conditions are surely violated); physical models must be constructed on the basis of meaningful solutions of the problems of magnetic hydrodynamics (or even better, kinetics). [43]

It is known $[17,41]$ that in non-ideal conductive media (plasmas) the magnetic flux conservation and the magnetic field line conservation properties are no longer equivalent, and the field line velocity $\boldsymbol{w}$ is not determined uniquely.

Hornig [20] considered this case in a purely geometric way and proved that the most general form of the induction equation preserving the magnetic field lines (magnetic topology) and not preserving the magnetic flux is as follows
}

$$
\boldsymbol{B}_{t}+\nabla \times(\boldsymbol{B} \times \boldsymbol{w})=\lambda \boldsymbol{B},
$$


$\boldsymbol{w}$ being the field line velocity, the component $\boldsymbol{w}_{\perp}$ of the field $\boldsymbol{w}$ not being uniquely determined, $\lambda$ being a scalar function of the fields $\boldsymbol{w}$ and $\boldsymbol{B}$.

We note that the equation (5.1) at $\boldsymbol{w}=\boldsymbol{u}$ directly follows from the second Zorawski criterion (1.8) applied to the vector field $\boldsymbol{a}(\boldsymbol{x}, t)=\boldsymbol{B}(\boldsymbol{x}, t)$ when accounting for the second equation of the system (2.3).

Kozlov [24] used even more general form of the condition for the vector field $\boldsymbol{a}(\boldsymbol{x}, t)$ to be material, $\boldsymbol{a}(\boldsymbol{x}, t), \boldsymbol{u}(\boldsymbol{x}, t): \mathbb{R}^{n+1} \rightarrow \mathbb{R}^{n}$, as follows

$$
\boldsymbol{a}_{t}+[\boldsymbol{a}, \boldsymbol{u}]=\lambda \boldsymbol{a},
$$

where $[\boldsymbol{a}, \boldsymbol{u}]$ is the commutator of the vector fields $\boldsymbol{a}(\boldsymbol{x}, t)$ and $\boldsymbol{u}(\boldsymbol{x}, t)$.

Preserving the magnetic topology means that the corresponding topological invariants of the filed lines, for example, knottedness, linkage etc., remain unaltered. Topological invariants of the field lines are explained in $[1,37]$.

\section{Magnetohydrodynamic Analogy}

The MHD analogy was originated by Batchelor [3] whose reasoning had been based on the well known fact that the equations for the vorticity in non-ideal fluids and for the magnetic induction (or for magnetic field) in non-ideal conductive media

$$
\begin{aligned}
& \dot{\boldsymbol{\Omega}}=\nabla \times(\boldsymbol{u} \times \boldsymbol{\Omega})+\nu \nabla^{2} \boldsymbol{\Omega}, \\
& \dot{\boldsymbol{B}}=\nabla \times(\boldsymbol{u} \times \boldsymbol{B})+\epsilon \nabla^{2} \boldsymbol{B},
\end{aligned}
$$

where $\nu$ and $\epsilon$ are the kinematic and the magnetic viscosities, are identical in form.

There is thus a formal analogy between the two solenoidal vectors $\boldsymbol{\Omega}$ and $\boldsymbol{H}$, provided $\boldsymbol{\Omega}$ refers to the motion of non-conducting fluid and $\boldsymbol{H}$ to the motion of conducting liquid.

Many of the results concerning vorticity in classical hydrodynamics can now be interpreted in terms of magnetic field in the electromagnetic hydrodynamic problem. [3]

The MHD analogy "is, in fact, an extension of the popular analogy between vorticity $\boldsymbol{\Omega}$ and material line elements $\boldsymbol{h}$ (proposed by Taylor 1938 [47], and which goes back to Helmholtz 1858 [19] and Kelvin 1880 [23]), equations for which in the absence of viscosity are identical in form as well" [19] (see the above equations (1.11), (4.3)):

$$
\begin{gathered}
\dot{\boldsymbol{\Omega}}=\boldsymbol{\Omega}_{t}+\boldsymbol{u} \cdot \nabla \boldsymbol{\Omega}=\boldsymbol{\Omega} \cdot \nabla \boldsymbol{u}, \\
\dot{\boldsymbol{h}}=\boldsymbol{h}_{t}+\boldsymbol{u} \cdot \nabla \boldsymbol{h}=\boldsymbol{h} \cdot \nabla \boldsymbol{u} .
\end{gathered}
$$

We note that it was surely Maxwell who first proposed the IMHD analogy. In the footnote at the last page of [31] he remarked the following.

Since the first part of this paper was written, I have seen in Crelle's Journal for 1859, a paper by Prof. Helmholtz on Fluid Motion, in which he has pointed out that the lines of fluid motion are arranged according to the same laws as the lines of magnetic force, the path of an electric current corresponding to a line of axes of those particles of the fluid which are in a state of rotation. This is an additional 
instance of a physical analogy, the investigation of which may illustrate both electro-magnetism and hydrodynamics. [30]

Later on he referred to the IMHD analogy, but as an assumption.

It is impossible, in our present state of ignorance as to the nature of the vortices, to assign the form of the law which connects the displacement of the medium with the variation of the vortices. We shall therefore assume that the variation of the vortices caused by the displacement of the medium is subject to the same conditions which Helmholtz, in his great memoir on Vortex-motion [19], has shewn to regulate the variation of the vortices of a perfect liquid. [36]

Nowadays these analogies are utilized in most of textbooks on HD and MHD, for example, the analogy between $\boldsymbol{\Omega}$ and $\boldsymbol{h}$ is considered to be valid in [27], though "the above analogies have since been realized to be flawed" [50].

Indeed, at the kinematic level, $\boldsymbol{\Omega}=\nabla \times \boldsymbol{u}$, whereas $\boldsymbol{B}=\nabla \times \boldsymbol{A}$, but the vector potential $\boldsymbol{A}$ is not present in the induction law for $\boldsymbol{B}$ (for both cases, the ideal and the non-ideal ones). At the dynamic level the differences between $\boldsymbol{\Omega}$ and $\boldsymbol{H}$ (or $\boldsymbol{B}$ ) are even more evident. One should address directly to the article of Tsinober [50] to find much more on the subject, including experimental evidence.

The current study explains the absence of the MHD analogy between $\boldsymbol{\Omega}$ and $\boldsymbol{H}$ (or $\boldsymbol{B}$ ) and some known flaws of the analogy, since the definition of the magnetic field $\boldsymbol{H}$ (3.1) given by Maxwell has nothing in common with the definition of the vorticity $\boldsymbol{\Omega}$ of a medium or the angular velocity $\boldsymbol{\omega}$ of quasi-rigid rotation of the vortex tubes of Faraday. And it is the Boltzmann correction to the magnetic induction law that explicitly accounts for the difference between $\boldsymbol{H}$ (or $\boldsymbol{B}$ ) and $\boldsymbol{\Omega}$.

\section{Conclusions}

1. The induction law after Minkowski is based on relativistic geometrical approach involving no physics of deformable media.

2. The induction law after Maxwell is fully based on the evident theory of molecular vortices but contradicts the definition of magnetic field by Maxwell.

3. The induction law after Boltzmann fixes faults of the Maxwell approach but implies the tubular foliation of the space filled with a deformable medium.

4. The induction law after Hornig involves an undetermined scalar function and looks as it were a more general case compared to the induction law after Boltzmann, nevertheless the former does not imply tubular foliation of the space.

\section{Acknowledgements}

Preliminaries of the current study were presented in [7-9]. The author would like to express his gratitude for those opportunities to the late Prof. M. S. Ivanov of Khristianovich Institute of Theoretical and Applied Mechanics, Siberian Branch of RAS (Novosibirsk, Russia), to Prof. A. P. Chupakhin and Prof. A. A. Chesnokov of Lavrientiev Institute of Hydrodynamics, Siberian Branch of RAS (Novosibirsk, Russia), and to Prof. Yu. D. Chashechkin of Institute for Problems in Mechanics, RAS (Moscow, Russia). 


\section{References}

1. V.I. Arnold, B. A. Khesin, Topological Methods in Hydrodynamics, Springer, New York, 1998.

2. T. W. BARRETT, Topological Foundations of Electromagnetism, World Scientific, Hackensack, 2008.

3. G. K. BATCHELOR, On the spontaneous magnetic field in a conducting liquid in turbulent motion, Proc. R. Soc. Lond. A, 201 (1950), 405-416.

4. L. Boltzmann, Über Faraday's Kraftlinien von Maxwell, Ostwalds Klassiker der Exacten Wissenschaften, No.69, W. Engelmann, Leipzig, 1895.

5. L. Boltzmann, Über Physikalische Kraftlinien, Ostwalds Klassiker der Exacten Wissenschaften, No. 102, W. Engelmann, Leipzig, 1898.

6. L. Boltzmann, Some errata in Maxwell's paper 'On Faraday's lines of force', Nature, 57 (1897), $77-79$.

7. V.L. BoRsch, The Faraday induction law after the Boltzmann correction and the problem of magnetic reconnection, in International Conference on Methods of Aerophysical Research, ICMAR 2010, Khristianovich Institute of Theoretical and Applied Mechanics, Siberian Branch of RAS, Novosibirsk, Russia, November 1-6, 2010.

8. V.L. Borsch, Derivation of the magnetic induction equation from the hypothesis of molecular vortices after Boltzmann, in Russian conference "Nonlinear Waves: Theory and New Applications", NW 2011, Lavrientiev Institute of Hydrodynamics, Siberian Branch of RAS, Novosibirsk, Russia, March 2-4, 2011.

9. V. L. BoRSCH, The original Maxwell electromagnetism as a fluent microcontinuum theory. A fresh look based on Boltzmann comments, in Colloquium EuroMech 531 "Vortices and Waves: Identifications and Mutual Influences", Lomonosov Moscow State University, Moscow, Russia, June 21-24, 2011.

10. J. CAT, One understanding: Maxwell on the methods of illustration and scientific metaphor, Stud. Hist. Mod. Phys., 32 (2001), 395-441.

11. O. Darrigol, The electrodynamics of moving bodies from Faraday to Hertz, Centaurus, 36 (1993), $245-360$.

12. O. DARrigol, Electrodynamics from Ampère to Einstein, Oxford University Press, Oxford, 2000.

13. O. Darrigol, Les Èquations de Maxwell: de MacCullagh à Lorentz, Belin, Paris, 2005.

14. P. A. Davidson, An Introduction to Magnetohydrodynamics, Cambridge University Press, Cambridge, 2001.

15. M. Faraday, Experimental researches in electricity. First series, Phil. Trans. R. Soc. Lond., 122 (1832), $126-162$.

16. J. P. Goedbloed, S. Poedts, Principles of Magnetohydrodynamics with Applications to Laboratory and Astrophysical Plasmas, Cambridge University Press, Cambridge, 2004.

17. J. P. Goedbloed, R. Keppens, S. Poedts, Advanced Magnetohydrodynamics with Applications to Laboratory and Astrophysical Plasmas, Cambridge University Press, Cambridge, 2010.

18. M. E. Gurtin, E. Fried, L. Anand, The Mechanics and Thermodynamics of Continua, Cambridge University Press, Cambridge, 2009.

19. H. Helmholtz, Über Integrale der Hydrodynamischen Gleichungen welche den Wirbelbewegungen Entsprechen, J. Reine Angew. Math., 55 (1858), 25-55. 
20. G. Hornig, K. Schindler, Magnetic topology and the problem of its invariant definition, Phys. Plasmas, 3 (1996), 781-791.

21. A. Jeffrey, Magnetohydrodynamics, Oliver \& Boyd, Edinburgh, 1966.

22. B. B. Kadomtsev, Magnetic field line reconnection, Rep. Progr. Phys., 50 (1987), $115-143$.

23. Lord Kelvin (W. Thomson), Vibration of columnar vortex, Phil. Mag., 10 (1880), $155-168$.

24. V.V. KozLov, The frozen-in condition for a direction field, small denominators and chaotization of steady flows of a viscous fluid, J. Appl. Math. Mech., 63 (1999), $229-235$.

25. H. LAmB, Hydrodynamics, Cambridge University Press, London, 1975.

26. K. LAmBert, The uses of analogy: James Clerk Maxwell's 'On Faraday's lines of force' and early Victorian analogical argument, British J. Hist. Sci., 44 (2011), $61-68$.

27. L. D. Landau, E. M. Lifshitz, Electrodynamics of Continuous Media, Pergamon Press, Oxford, 1984.

28. H. A. Lorentz, The Theory of Electrons and its Applications to the Phenomena of Light and Radiant Heat, Teubner, Leipzig, 1916.

29. J. C. Maxwell, On Faraday's lines of force, Trans. Camb. Phil. Soc., 10 (1864), 27-83. (Part I read 1855, part II read 1856.)

30. J. C. MAXwell, On physical lines of force. Part I. The theory of molecular vortices applied to magnetic phenomena, Phil. Mag., 21 (1861), 161-175.

31. J. C. MAXwell, On physical lines of force. Part II. The theory of molecular vortices applied to electric currents, Phil. Mag., 21 (1861), 281-291, 338-348.

32. J. C. Maxwell, On physical lines of force. Part III. The theory of molecular vortices applied to statical electricity, Phil. Mag., 23 (1862), 12-24.

33. J. C. Maxwell, On physical lines of force. Part IV. The theory of molecular vortices applied to the action of magnetism on polarized light, Phil. Mag., 23 (1862), $85-95$.

34. Maxwell J. C., A Dynamical Theory of the Electromagnetic Field, Phil. Trans. R. Soc. Lond., CLV (1865), 459-512.

35. J. C. Maxwell, A Treatise on Electricity and Magnetism, Vol. I, Clarendon Press, Oxford, 1873.

36. J. C. Maxwell, A Treatise on Electricity and Magnetism, Vol. II, Clarendon Press, Oxford, 1873.

37. H. K. Moffatt, Magnetic Field Generation in Electrically Conducting Fluids, Cambridge University Press, Cambridge, 1978.

38. K. Moffatt, Vortex Dynamics: The legacy of Helmholtz and Kelvin, in A. V. BoRISOV ET AL. (eds.), IUTAM Symposium on Hamiltonian Dynamics, Vortex Structures, and Turbulence, Steklov Mathematical Institute, RAS, Moscow, August 25-30, 2006, Springer, Dordrecht, 2008, 1-10.

39. N. J. NERSESSIAN, Maxwell and 'The method of physical analogy': model-based reasoning, generic abstraction, and conceptual change, in D. B. MALAMENT (ed.), Reading Natural Philosophy: Essays in the History and Philosophy of Science and Mathematics, Open Court, Chicago, 2002, 129-166.

40. W. Pauli, Theory of Relativity, Pergamon Press, London, 1958.

41. E. Priest, T. Forbes, Magnetic Reconnection: MHD Theory and Aplications, Cambridge University Press, Cambridge, 2000. 
42. R. Prim, C. Truesdell, A derivation of Zorawski's criterion for permanent vector-lines, Proc. AMS, 1 (1950), 32-34.

43. M. I. Pudovkin, V.S. Semenov, Magnetic field reconnection theory and the solar wind-magnetosphere interaction: A review, Space Sci. Rev., 41 (1985), 1-89.

44. L. I. Sedov, On the Ponderomotive Forces of Interaction of Electromagnetic Field and an Accelerating Material Continuum, taking into Account Finite Deformations, J. Appl. Math. Mech., 29 (1965), 4-17.

45. L. I. SEDov, On the Addition of Motions Relative to Deformable Reference Systems, J. Appl. Math. Mech., 42 (1978), 181-184.

46. D. M. SiEgEL, Innovation in Maxwell's Electromagnetic Theory: Molecular Vortices, Displacement Current, and Light, Cambridge University Press, Cambridge, 1991.

47. G. I. TAYLOR, Production and dissipation of vorticity in a turbulent fluid, Proc. R. Soc. Lond. A, 164 (1938), 15-23.

48. C. TRUESDELL, The kinematics of vorticity, Indiana University Press, Bloomington, 1954.

49. C. Truesdell, R. A. Toupin, The classical fields theories. Principles of classical mechanics and field theories, in S. FLUGGE (ed.), Encyclopedia of Physics, Vol. III / 1, Springer, Berlin, 1960, 226-793.

50. A. TSINOBER, How analogous is generation of vorticity and passive vectors (magnetic fields)?, in S. MoLOKOV ET AL. (eds), Magnetohydrodynamics: Historical Evolution and Trends, Springer, Dordrecht, 2007, 223-230.

51. M. Yamada, R. Kulsrud, H. Ji, Magnetic Reconnection, Rev. Mod. Phys., 82 (2010), 603-664.

52. E. G. Zweibel, M. Yamada, Perspectives on magnetic reconnection, Proc. R. Soc. Lond. A, 472 (2016), $1-30$.

53. K. Zorawski, Über die Erhaltung der Wirbelbewegung, C. R. Acad. Sci. Cracovie, $1900,335-341$. 